\title{
Estudio etnobotánico de plantas medicinales utilizadas en comunidades adyacentes al Área de Conservación Privada San Antonio, Chachapoyas, Amazonas, Perú.
}

\section{Ethnobotanical study of medicinal plants used in communities adjacent to San Antonio Private Conservation Area, Chachapoyas, Amazonas, Peru.}

\author{
*Eder A. Chilquillo Torres ${ }^{1,2}$, Joaquina Albán ${ }^{3}$ y Aldo Muñoz ${ }^{4}$
}

\section{RESUMEN}

Se presenta un inventario etnomedicinal y un análisis de su importancia en las comunidades adyacentes al Área de Conservación Privada San Antonio, Chachapoyas, Amazonas, Perú, con la finalidad de contribuir al rescate y valoración del conocimiento tradicional sobre el uso de recursos. Cuatro comunidades fueron estudiadas: San Antonio, Pencapampa, Lumac Urco y Quipachacha. Treinta y seis personas de ambos sexos con edades que oscilan entre 20-75 años participaron de la investigación. Se determinó las plantas medicinales de mayor importancia relativa (IR), los trastornos médicos más citados entre los pobladores (FCI) y se estimó el nivel de conocimiento cultural en cada comunidad estudiada (índice H). Se reportaron 124 especies etnomedicinales pertenecientes a 104 géneros y 47 familias taxonómicas siendo Asteraceae y Lamiaceae las más representativas. Dentro de las especies más importantes se destacan: "hierba santa" Cestrum auriculatum L'Hér., "lancetilla" Alternanthera porrigens (Jacq.) Kuntze y "pie de perro" Desmodium uncinatum (Jacq.) DC. Entre las afecciones médicas generalmente tratadas con plantas medicinales se destacan: trastornos genito-urinario $(\mathrm{FCI}=0.65)$, trastornos respiratorios $(\mathrm{FCI}=0.65)$ y trastornos digestivos $(\mathrm{FCI}=0.55)$. Finalmente, los altos valores de $\mathrm{H}^{\prime}(4.59-$ 5.5 ) encontrados en las comunidades estudiadas nos demuestran el alto nivel de conocimiento etnomedicinal que mantienen sus pobladores, lo cual emerge como una ventana promisoria para posteriores estudios de conservación de recursos y manejo sostenible.

Palabras clave: conocimientos tradicionales, conservación, etnobotánica, erosión cultural.

\begin{abstract}
An ethnomedicinal inventory and an analysis of their importance in the communities adjacent to the San Antonio Private Conservation Area, Chachapoyas, Amazonas, Peru, is presented here, with the purpose of contributing to the rescue and valuation of traditional knowledge about the use of resources. Four communities were studied: San Antonio, Pencapampa, Lumac Urco and Quipachacha. Thirty-six people of both sexes aged between 20 - 75 years were interviewed individually in order to collect ethnomedicinal information. It was determined the medicinal plants of greater relative importance (RI), the medical conditions most cited among the inhabitants (ICF) and the level of cultural knowledge was estimated in each community studied ( $\mathrm{H}$ index). A total of 124 ethnomedicinal species belonging to 104 genera and 47 taxonomic families were reported, with Asteraceae and Lamiaceae being the most representative. Among the most important species were: "hierba santa" Cestrum auriculatum L'Hér., "Lancetilla" Alternanthera porrigens (Jacq.) Kuntze and "pie de perro" Desmodium uncinatum (Jacq.) DC. Among the medical conditions that usually are reported for treatment with medicinal plants were: genito-urinary disorders $(\mathrm{CRF}=0.65)$, respiratory disorders $(\mathrm{CRF}=0.65)$ and digestive disorders $(\mathrm{CRF}=0.55)$. Finally, the high values of the $\mathrm{H}$ index $(4.59-5.5)$ found in the communities studied show us the high level of ethnomedicinal knowledge maintained by its inhabitants, which emerges as a promising window for further studies of resource conservation and sustainable management.
\end{abstract}

Keywords: traditional knowledge, conservation, ethnobotany, cultural erosion. ${ }^{1}$

${ }^{1}$ Programa de Pós-Graduação em Biologia Vegetal, Instituto de Biologia, Universidade Estadual de Campinas - UNICAMP, CP. 6109, 13083970, Campinas, SP, Brasil. (*) Email: e.chilquillo.torres@gmail.com

${ }^{2}$ Departamento de Etnobotánica y Botánica Económica - Museo de Historia Natural, Universidad Nacional Mayor de San Marcos, Av.

Arenales 1256, Jesús María, Lima, Perú.

${ }^{3}$ Área de Conservación Privada San Antonio - Jr. Chincha Alta Nº569, Chachapoyas, Perú. 


\section{INTRODUCCIÓN}

El Perú, es considerado entre los diecisiete países más diversos del mundo (Fajardo, Lessmann, Bonaccorso, Devenish, \& Muñoz, 2014) albergando ca. 19500 especies vegetales, las cuales representan el 10\% de la diversidad global de plantas (Gentry, 1982; Brako \& Zarucchi, 1993). Históricamente, esta amplia diversidad vegetal ha servido al poblador peruano como fuente primaria de alimentos, medicina, energía, construcción, fibras, actividades humanas simbólicas y ritualistas (De-la-Cruz, Vilcapoma, \& Zevallos, 2007) permitiendo de esta manera la acumulación de conocimientos tradicionales asociado a especies vegetales.

Los conocimientos tradicionales representan una acumulación detallada y sistematizada de observaciones de uso de la biodiversidad (Berkes et al., 2000) propio de cada grupo cultural (Boster, 1986; Atran etal., 2002; Torre etal., 2006), los cuales en la actualidad vienen sufriendo fuertes procesos de aculturación producto de las migraciones, acceso a nuevas tecnologías y destrucción de hábitats producto de actividades extractivas (Case et al., 2005; Reyes-García etal., 2006).

Los estudios etnobotánicos en tal sentido, establecen un puente de comprensión cultural a través de una perspectiva científica acerca del desenvolvimiento de estrategias y mecanismos de regulación del uso de recursos (Phillips etal., 1994; Lawrence etal., 2005). De esta manera, evaluaciones etnomedicinales resultan importantes para su conservación, protección y bioprospección (Balick, 1990; McClatchey, Mahady, Bennett, Shiels, \& Savo, 2009).

La región Amazonas, situada en norte del territorio peruano constituye un mosaico de zonas de vidas andinas, las cuales albergan una biodiversidad única (Encarnación \& Zárate, 2010), la cual es ampliamente usada por etnias ancestrales y campesinas caracterizadas por difundido conocimiento agroecológico y cultural (Grados Vasquez \& Peláez, 2014). Sin embargo, debido a expansión agrícola, deforestación y el desarrollo de minería varias hectáreas de bosque vienen siendo amenazados (Garcia, 2008). Por lo que, numerosas propuestas de Áreas de Conservación Privada (ACP) vienen constituyéndose y tornándose efectivas en el tiempo(SERNARP, 2014), como es el caso del ACP San Antonio, la cual tiene como finalidad salvaguardar los últimos bosques de montaña situados de forma aledaña a la ciudad de Chachapoyas y promover el uso sostenible de sus recursos.
El objetivo de este estudio es documentar el conocimiento tradicional asociado a plantas medicinales y analizar su importancia en la vida diaria de los pobladores de las comunidades adyacentes al ACP San Antonio.

\section{MATERIAL Y MÉTODOS}

\section{Área de estudio}

La investigación fue realizada en 04 comunidades campesinas: San Antonio, Pencapampa, Lumac Urco y Quipachacha, las cuales se encuentran limítrofes al ACP San Antonio (Figura 1). Esta área de conservación se encuentra ubicado en el departamento de Amazonas, provincia de Chachapoyas y su extensión es de 3573900 ha., caracterizados por la presencia de relictos de bosques montanos.

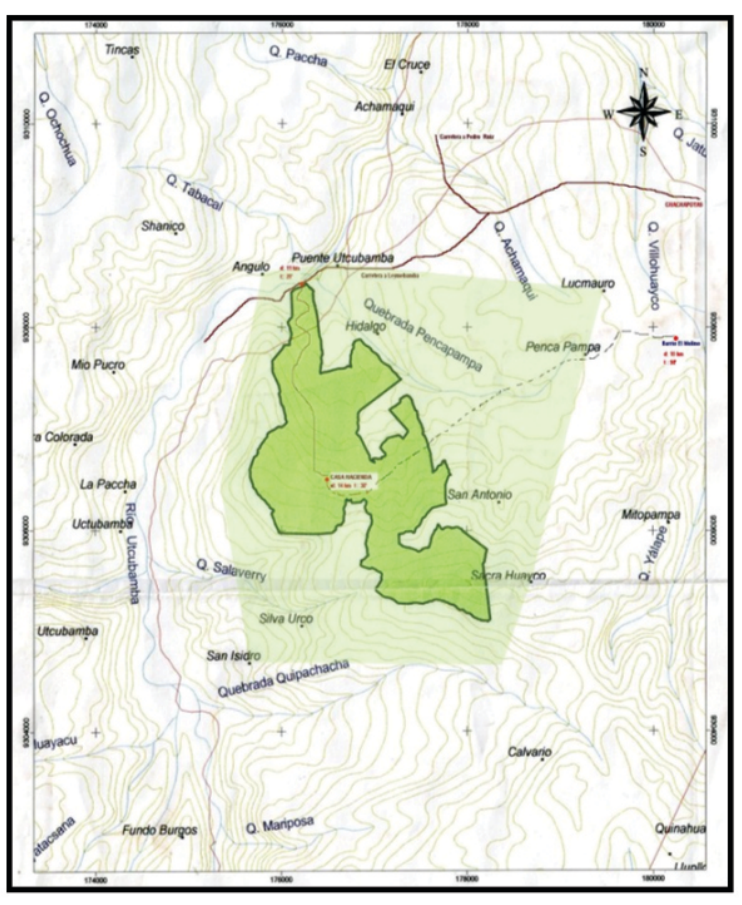

Figura 1. Localización del ACP San Antonio y los poblados aledaños (Fuente: ACP San Antonio).

\section{Diseño y recolección de información etnobotánica}

Inicialmente se recopiló información etnográfica, socio-económica y florística asociada a la zona de estudio, con la finalidad de obtener un panorama general de los lugares donde se realizará la investigación. Posteriormente se dialogó con las autoridades del ACP San Antonio y las comunidades que formaron parte de la investigación (Figura 2). Se utilizó la técnica de "snow ball", la cual consiste en ubicar "personas claves" dentro de las comunidades (Bernard, 2006; Christo et al., 2006) con la finalidad 
de tener un primer acercamiento a la población. Se utilizaron entrevistas estructuradas siguiendo los lineamientos propuestos por Albán (1998). Las entrevistas fueron realizadas en el mes de febreromarzo del 2009, considerándose pobladores de ambos sexos que estuvieran en un rango de edad de 22 a 75 años.

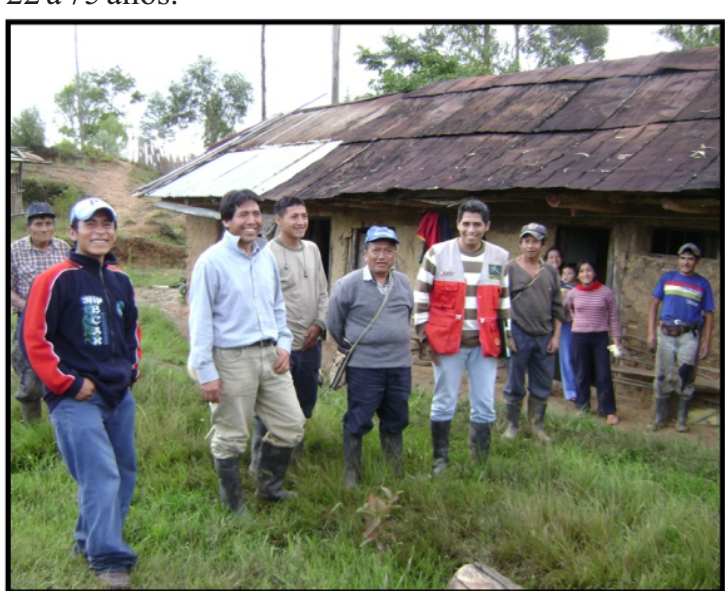

Figura 2. Reuniones participativas con autoridades de las comunidades en estudio

\section{Recolección, preservación y determinación de especímenes botánicos}

Se empleó el método tradicional de colecta y preservación de muestras botánicas propuesto por Cerrate (1969) (Figura 3). Los ejemplares botánicos fueron depositados en el Herbario San Marcos (USM). Se utilizó bibliografía especializada para las determinaciones botánicas. El ordenamiento taxonómico de las especies, siguió la propuesta de Angiosperm Phylogeny Group (APG., 1998; APG II., 2003; APG III., 2009; APG IV., 2016).

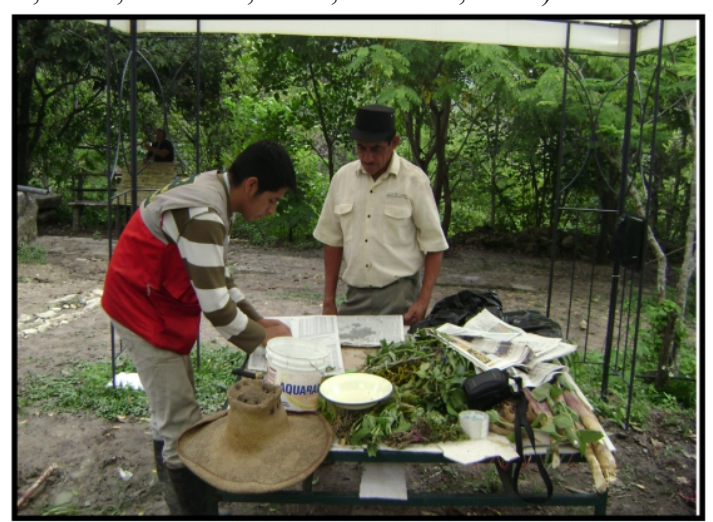

Figura 3. Prensado de ejemplares botánicos

Ordenamiento y Sistematización de datos etnobotánicos

Los diversos usos medicinales fueron sistematizados en 12 categorías de dolencia (Tabla 1) propuestas por la organización mundial de salud (WHO, 2016).
Tabla 1. Categorías de dolencia medicinal propuestas por laWHO (2016)

\begin{tabular}{|l|}
\hline Sub-categorías medicinales (WHO, 2016) \\
\hline Afecciones cutáneas y subcutáneas \\
Trastornos de los ojos y anexos \\
Trastornos del oído y procesos mastoideos \\
Trastornos del sistema circulatorio \\
Trastornos del sistema digestivo \\
Trastornos del sistema genito-urinario \\
Trastornos del sistema musculo esquelético \\
Trastornos del sistema nervioso \\
Trastornos del sistema respiratorio \\
Trastornos endocrinos, nutricionales y metabólicas \\
Dolencias infecciosas y parasitarias \\
Síntomas y afecciones clínicamente no clasificados
\end{tabular}

\section{Cuantificación de la información etnobotánica}

Con la finalidad de cuantificar la información recabada y hacerla comparable en el tiempo, se utilizaron los siguientes índices

Índice de Importancia Relativa (Bennett \& Prance, 2000), determina la especie más importante.

$$
\mathrm{RI}=\mathrm{NUC}+\mathrm{NT}
$$

Donde:

NUC: numero de categorías de dolencia para una especie dada dividido por el número total de categorías de dolencia de la especie más versátil. NT: numero de usos atribuido a una especie dada dividido por el número total de usos de la especie más versátil.

Factor de Consenso de Informantes (FCI) (Trotter \& Logan, 1986), estima la importancia de las diferentes especies para una determinada categoría de dolencia.

$$
\mathrm{FCI}=\mathrm{NAR}-\mathrm{NT} / \mathrm{NAR}-1
$$

Donde:

NAR: sumatoria de usos registrados por cada informante para una categoría

NT: número de especies indicadas en cada categoría

Índice de Shannon (Begossi, 1996), determina el nivel de conocimiento de cada comunidad estudiada.

$$
\mathrm{H}^{\prime}=\sum_{i=i}^{S}(\mathrm{pi})(\log p i)
$$

Dónde: $\mathrm{pi}=\mathrm{ni} / \mathrm{N}$

$\mathrm{s}$ : número de especies

ni: número de citaciones por especie

$\mathrm{N}$ : número total de citaciones 


\section{RESULTADOS Y DISCUSIÓN}

En total, 124 especies vegetales pertenecientes a 104 géneros y 47 familias taxonómicas de plantas vasculares fueron reportadas como etnomedicinales en las comunidades estudiadas (Tabla 2) (Figura 4). Asteraceae fue la familia con mayor número de especies medicinales (18), seguido por Lamiaceae (12), Fabaceae (10) y Solanaceae (8), juntas constituyen el $38.7 \%$ del total de las especies medicinales reportadas. Nuestros resultados en términos de riqueza de especies etnomedicinal resultan concordantes con otros estudios llevados en eco-regiones del norte del Perú (Alva, Quezada, \& Rodríguez, 2016; Bussmann \& Sharon, 2006; Monigatti, Bussmann, \& Weckerle, 2013).

Por otro lado, cabe resaltar que el $80 \%$ del total de especies etnomedicinales está compuesto por vegetación herbácea, lo cual lo convierte en un componente frágil ante la quema de áreas con fines agrícolas e incendios forestales observados en la zona de estudio. El 31\% la flora etnomedicinal está compuesta por especies introducidas, donde el $98 \%$ es cultivada en las comunidades estudiadas, lo cual demuestra el rol preponderante de las plantas introducidas en la manutención de la salud de los pobladores(Bennett \& Prance, 2000).

Tabla 2. Listados especies medicinales reportados en comunidades adyacentes al ACP San Antonio

\begin{tabular}{|c|c|c|c|c|c|c|c|c|}
\hline Familia taxonómica & Nombre científico & Nombre vernácular & Porte & Origen & Domesticación & $\begin{array}{c}\text { Categoría } \\
\text { medicinal }(*)\end{array}$ & Parte usada & $\begin{array}{c}\text { Forma de } \\
\text { preparación }\end{array}$ \\
\hline Adoxaceae & $\begin{array}{l}\text { Sambucus peruviana Kunth } \\
\text { Alternanthera porrigens (Jacq.) }\end{array}$ & Sauco & Arbóreo & Nativo & Cultivado & $\mathrm{Di}, \mathrm{Mu}, \mathrm{Pa}$ & $\begin{array}{l}\text { Tallo } \\
\text { Planta }\end{array}$ & Infusión \\
\hline Amaranthaceae & Kuntze & Lancetilla & Herbáceo & Nativo & Silvestre & $\mathrm{Ge}, \mathrm{Mu}, \mathrm{En}$ & entera & Infusión \\
\hline Amaranthaceae & Chenopodium ambrosioides $\mathrm{L}$. & Paico & Herbáceo & Nativo & Silvestre & $\mathrm{Di}, \mathrm{Ne}, \mathrm{Pi}$ & Hoja & Infusión \\
\hline Anacardiaceae & Schinus molle L. & Molli & Arbóreo & Nativo & Silvestre & $\mathrm{Mu}$ & Hoja & Infusión \\
\hline Apiaceae & Pimpinella anisum $\mathrm{L}$. & Anis & Herbáceo & Introducido & Cultivado & $\mathrm{Di}$ & Hoja & Infusión \\
\hline Apiaceae & Foeniculum vulgare P. Miller & Hinojo & Herbáceo & Introducido & Silvestre & $\mathrm{Di}$ & Hoja & Infusión \\
\hline Apiaceae & $\begin{array}{l}\text { Arracacia xanthorrhiza Bancr. } \\
\text { Daucus montanus Humb. \& }\end{array}$ & $\begin{array}{l}\text { Racacha del abuelo } \\
\text { Zanahoria del }\end{array}$ & Herbáceo & Nativo & Silvestre & Nn & $\begin{array}{l}\text { Raíz } \\
\text { Planta }\end{array}$ & Infusión \\
\hline Apiaceae & Bonpl. ex Spreng. & abuelo & Herbáceo & Nativo & Silvestre & $\mathrm{Nn}$ & entera & Infusión \\
\hline Apocynaceae & Couma macrocarpa Barb. Rodr. & Leche caspe & Arbóreo & Nativo & Silvestre & $\mathrm{Nn}$ & Tallo & Decocción \\
\hline Arecaceae & Arecaceae sp. & Palma & Herbáceo & Nativo & Silvestre & $\mathrm{Pi}$ & Fruto & $\begin{array}{l}\text { Decocción } \\
\text { Decocción, }\end{array}$ \\
\hline Asphodelaceae & Aloe vera (L.) Burm. f. & Penca sabila & Herbáceo & Introducido & Silvestre & $\mathrm{Ci}, \mathrm{Ge}, \mathrm{Mu}, \mathrm{Pi}, \mathrm{Nn}$ & $\begin{array}{l}\text { Hoja } \\
\text { Planta }\end{array}$ & emplasto \\
\hline Asteraceae & Cynara scolymus $\mathrm{L}$. & Alcachofa & Herbáceo & Introducido & Cultivado & $\mathrm{Ci}$ & entera & Decocción \\
\hline Asteraceae & $\begin{array}{l}\text { Tagetes pusilla Kunth } \\
\text { Schkuhria pinnata (Lam.) Kuntze }\end{array}$ & Anis de monte & Herbáceo & Nativo & Silvestre & $\mathrm{Di}$ & $\begin{array}{l}\text { Hoja } \\
\text { Planta }\end{array}$ & Infusión \\
\hline Asteraceae & $\begin{array}{l}\text { ex Thell. } \\
\text { Hypochaeris taraxacoides (Walp.) }\end{array}$ & Canchalagua & Herbáceo & Nativo & Silvestre & $\mathrm{Ci}$ & $\begin{array}{l}\text { entera } \\
\text { Planta }\end{array}$ & Infusión \\
\hline Asteraceae & Ball & Chicoria & Herbáceo & Nativo & Silvestre & $\mathrm{Ge}, \mathrm{Pa}, \mathrm{En}$ & $\begin{array}{l}\text { entera } \\
\text { Planta }\end{array}$ & Infusión \\
\hline Asteraceae & $\begin{array}{l}\text { Baccharis tricuneata (L. f.) Pers. } \\
\text { Baccharis latifolia (Ruiz \& Pav.) }\end{array}$ & Chishka & Herbáceo & Nativo & Silvestre & $\mathrm{Re}, \mathrm{Di}, \mathrm{Ge}, \mathrm{Mu}, \mathrm{Oi}$ & entera & Infusión \\
\hline Asteraceae & $\begin{array}{l}\text { Pers. } \\
\text { Senecio aff. canescens (Bonpl.) }\end{array}$ & Chishka brava & Herbáceo & Nativo & Silvestre & $\mathrm{Di}, \mathrm{Mu}$ & Hoja & Infusión \\
\hline Asteraceae & Cuatrec. & Cira cira & Herbáceo & Nativo & Silvestre & $\mathrm{Re}, \mathrm{Nn}$ & Hoja & Infusión \\
\hline Asteraceae & $\begin{array}{l}\text { Cosmos sp. } \\
\text { Acanthoxanthium spinosum (L.) }\end{array}$ & Cosmo & Herbáceo & Introducido & Silvestre & $\mathrm{Pa}$ & $\begin{array}{l}\text { Hoja } \\
\text { Planta }\end{array}$ & Infusión \\
\hline Asteraceae & Fourr. & Juan alonso & Herbáceo & Nativo & Silvestre & $\mathrm{Ci}$ & $\begin{array}{l}\text { entera } \\
\text { Planta }\end{array}$ & Infusión \\
\hline Asteraceae & Matricaria recutita $\mathrm{L}$. & Kallu manzanilla & Herbáceo & Introducido & Silvestre & $\mathrm{Re}, \mathrm{Pa}, \mathrm{Vi}, \mathrm{Nn}$ & $\begin{array}{l}\text { entera } \\
\text { Planta }\end{array}$ & Infusión \\
\hline Asteraceae & Sonchus oleraceus $\mathrm{L}$. & Kasha cerraja & Herbáceo & Nativo & Silvestre & $\mathrm{Re}, \mathrm{Di}$ & $\begin{array}{l}\text { entera } \\
\text { Planta }\end{array}$ & Infusión \\
\hline Asteraceae & Gnaphalium spicatum Mill. & Lechuguilla & Herbáceo & Nativo & Silvestre & $\mathrm{Ge}, \mathrm{Vi}$ & $\begin{array}{l}\text { entera } \\
\text { Planta }\end{array}$ & Infusión \\
\hline Asteraceae & Matricaria chamomilla $\mathrm{L}$. & Manzanilla & Herbáceo & Nativo & Cultivado & $\mathrm{Di}, \mathrm{Vi}$ & $\begin{array}{l}\text { entera } \\
\text { Planta }\end{array}$ & Infusión \\
\hline Asteraceae & Tagetes multiflora Kunth & Maria sacha & Herbáceo & Nativo & Silvestre & $\mathrm{Nn}$ & $\begin{array}{l}\text { entera } \\
\text { Planta }\end{array}$ & $\begin{array}{l}\text { Infusión } \\
\text { Infusión, }\end{array}$ \\
\hline Asteraceae & Ambrosia peruviana All. & Marko & Herbáceo & Nativo & Silvestre & $\operatorname{Re}$ & entera & Vapor \\
\hline Asteraceae & $\begin{array}{l}\text { Tessaria integrifolia } \text { Ruiz \& Pav. } \\
\text { Parastrephia aff. quadrangularis }\end{array}$ & Pajaro bobo & Herbáceo & Nativo & Silvestre & $\mathrm{Ge}, \mathrm{En}$ & $\begin{array}{l}\text { Hoja } \\
\text { Planta }\end{array}$ & Infusión \\
\hline Asteraceae & $\begin{array}{l}\text { (Meyen) Cabrera } \\
\text { Baccharis genistelloides (Lam.) }\end{array}$ & Tola & Herbáceo & Nativo & Silvestre & $\mathrm{Ci}, \mathrm{Pi}$ & entera & Infusión \\
\hline Asteraceae & Pers. & Tres esquinas & Herbáceo & Nativo & Silvestre & $\mathrm{En}, \mathrm{Nn}$ & Hoja & Infusión \\
\hline Basellaceae & Ullucus tuberosus Caldas & Olluco blanco & Herbáceo & Nativo & Cultivado & $\mathrm{Ge}$ & Raiz & Decocción \\
\hline Betulaceae & Alnus acuminata Kunth & Aliso & Arbóreo & Nativo & Silvestre & $\mathrm{Nn}$ & Tallo & Decocción \\
\hline Boraginaceae & $\begin{array}{l}\text { Borago officinalis } \mathrm{L} \text {. } \\
\text { Rorippa nasturtium-aquaticum }\end{array}$ & Borraja & Herbáceo & Nativo & Silvestre & $\operatorname{Re}$ & $\begin{array}{l}\text { Hoja } \\
\text { Planta }\end{array}$ & Infusión \\
\hline Brassicaceae & (L.) Hayek & Berro & Herbáceo & Introducido & Cultivado & $\mathrm{Ge}$ & $\begin{array}{l}\text { entera } \\
\text { Planta }\end{array}$ & Infusión \\
\hline Brassicaceae & Brassica oleracea $\mathrm{L}$. & $\mathrm{Col}$ & Herbáceo & Introducido & Cultivado & $\mathrm{Di}, \mathrm{Vi}$ & $\begin{array}{l}\text { entera } \\
\text { Planta }\end{array}$ & Infusión \\
\hline Brassicaceae & Brassica alba (L.) Rabenh. & Mostaza & Herbáceo & Introducido & Cultivado & $\operatorname{Re}$ & $\begin{array}{l}\text { entera } \\
\text { Planta }\end{array}$ & Infusión \\
\hline Campanulaceae & Lobelia tenera Kunth & San juanillo & Herbáceo & Nativo & Silvestre & Nn & entera & Infusión \\
\hline Cucurbitaceae & Cucurbita sp. & Calabaza & Herbáceo & Nativo & Cultivado & $\mathrm{Nn}$ & Hoja & Infusión \\
\hline Cucurbitaceae & Cyclanthera pedata (L.) Schrad. & Caygua & Herbáceo & Introducido & Cultivado & $\mathrm{Ci}, \mathrm{Oi}$ & Fruto & Decocción \\
\hline Cucurbitaceae & Sicyos edulis Jacq. & Chiclayo & Herbáceo & Nativo & Cultivado & $\mathrm{Pa}$ & Hoja & Infusión \\
\hline Cucurbitaceae & Sicyos baderoa Hook. \& Arn. & Habillas & Herbáceo & Nativo & Silvestre & $\mathrm{Pi}$ & Hoja & Infusión \\
\hline Cucurbitaceae & Cucumis sativus $\mathrm{L}$. & Pepino & Herbáceo & Nativo & Cultivado & $\mathrm{Re}, \mathrm{Ci}$ & Fruto & Decocción \\
\hline
\end{tabular}




\begin{tabular}{|c|c|c|c|c|c|c|c|c|}
\hline Equisetaceae & Equisetum giganteum $\mathrm{L}$. & Cola de caballo & Herbáceo & Introducido & Silvestre & $\mathrm{Ge}, \mathrm{En}$ & $\begin{array}{l}\text { Planta } \\
\text { entera }\end{array}$ & Infusión \\
\hline Erythroxylaceae & Erythroxylum coca $\mathrm{Lam}$. & Coca & Arbustivo & Nativo & Cultivado & $\mathrm{Di}, \mathrm{Nn}$ & Hoja & Infusión \\
\hline Euphorbiaceae & Ricinus communis $\mathrm{L}$. & Higuerilla & Herbáceo & Nativo & Silvestre & $\mathrm{Di}, \mathrm{Nn}$ & Semilla & Decocción \\
\hline Euphorbiaceae & $\begin{array}{l}\text { Ricinus sp. } \\
\text { Chamaesyce hypericifolia (L.) }\end{array}$ & Higuerilla blanca & Herbáceo & Introducido & Silvestre & $\mathrm{Di}, \mathrm{Ge}$ & $\begin{array}{c}\text { Semilla } \\
\text { Planta }\end{array}$ & Decocción \\
\hline Euphorbiaceae & Millspaugh & Leche leche & Herbáceo & Nativo & Silvestre & $\mathrm{Pa}, \mathrm{Nn}$ & entera & Infusión \\
\hline Euphorbiaceae & Manihot esculenta Crantz & Yuca cabalonga & Herbáceo & Introducido & Silvestre & $\mathrm{Nn}$ & Raiz & Decocción \\
\hline Fabaceae & $\begin{array}{l}\text { Lupinus mutabilis Sweet } \\
\text { Otholobium pubescens (Poir.) }\end{array}$ & Chocho del abuelo & Herbáceo & Nativo & Silvestre & $\mathrm{Nn}$ & Semilla & Decocción \\
\hline Fabaceae & $\begin{array}{l}\text { J.W. Grimes } \\
\text { Otholobium mexicanum (L.f.) }\end{array}$ & Culen & Herbáceo & Nativo & Silvestre & $\mathrm{Di}, \mathrm{Pa}$ & Hoja & Infusión \\
\hline Fabaceae & Grimes & Culencillo & Herbáceo & Nativo & Silvestre & $\mathrm{Di}, \mathrm{Vi}$ & Hoja & Infusión \\
\hline Fabaceae & Inga edulis $\mathrm{C}$. Martius & Guabo & Herbáceo & Nativo & Cultivado & $\mathrm{Di}$ & Fruto & Decocción \\
\hline Fabaceae & Erythrina edulis Triana ex Micheli & Pajur & Arbóreo & Nativo & Silvestre & $\mathrm{Di}, \mathrm{Ge}, \mathrm{Pa}, \mathrm{Nn}$ & Hoja & Decocción \\
\hline Fabaceae & $\begin{array}{l}\text { Phaseolus lunatus L. } \\
\text { Desmodium uncinatum (Jacq.) }\end{array}$ & Pallar & Herbáceo & Nativo & Cultivado & $\mathrm{Pi}$ & $\begin{array}{c}\text { Semilla } \\
\text { Planta }\end{array}$ & Decocción \\
\hline Fabaceae & DC. & Pie de perro & Herbáceo & Nativo & Silvestre & $\mathrm{Re}, \mathrm{Ci}, \mathrm{Ge}, \mathrm{En}$ & entera & Infusión \\
\hline Fabaceae & $\begin{array}{l}\text { Spartium junceum } \mathrm{L} \text {. } \\
\text { Caesalpinia spinosa (Molina) }\end{array}$ & Retama & Herbáceo & Nativo & Silvestre & $\mathrm{Re}, \mathrm{Di}, \mathrm{Pa}$ & Hoja & Infusión \\
\hline Fabaceae & Kuntze & Talla & Arbustivo & Nativo & Silvestre & $\operatorname{Re}$ & Fruto & Decocción \\
\hline Fabaceae & Senna sp. & Yutuy & Herbáceo & Nativo & Silvestre & $\mathrm{Nn}$ & Hoja & Infusión \\
\hline Juglandaceae & Juglans neotropica Diels & Nogal & Arbóreo & Introducido & Silvestre & $\mathrm{Mu}$ & $\begin{array}{l}\text { Hoja } \\
\text { Planta }\end{array}$ & Infusión \\
\hline Lamiaceae & Ocimum basilicum $\mathrm{L}$. & Albahaca & Herbáceo & Introducido & Cultivado & $\mathrm{Ge}, \mathrm{Nn}$ & entera & Infusión \\
\hline Lamiaceae & Hyptis capitata Jacq. & Cadillo & Herbáceo & Nativo & Silvestre & $\mathrm{Ge}$ & Hoja & Infusión \\
\hline Lamiaceae & $\begin{array}{l}\text { Hyptis eriocephala Benth. } \\
\text { Minthostachys mollis (Benth.) }\end{array}$ & Chispa sacha & Herbáceo & Introducido & Silvestre & $\mathrm{Nn}$ & Hoja & Infusión \\
\hline Lamiaceae & Griseb. & Cruz sacha & Herbáceo & Nativo & Silvestre & $\mathrm{Ci}, \mathrm{Nn}$ & Hoja & Infusión \\
\hline Lamiaceae & Mentha spicata $\mathrm{L}$. & Hierba buena & Herbáceo & Introducido & Cultivado & $\mathrm{Di}, \mathrm{Vi}$ & Hoja & Infusión \\
\hline Lamiaceae & Mentha $\times$ piperita $\mathrm{L}$. & Menta & Herbáceo & Introducido & Cultivado & $\mathrm{Re}, \mathrm{Di}$ & Hoja & Infusión \\
\hline Lamiaceae & Origanum majorana $\mathrm{L}$. & Oregano de monte & Herbáceo & Introducido & Silvestre & $\mathrm{Di}, \mathrm{Ge}$ & Hoja & Infusión \\
\hline Lamiaceae & Mentha sp. & Poleo & Herbáceo & Nativo & Silvestre & $\mathrm{Di}, \mathrm{Mu}, \mathrm{Nn}$ & Hoja & Infusión \\
\hline Lamiaceae & Rosmarinus officinalis $\mathrm{L}$. & Romero & Herbáceo & Nativo & Silvestre & $\mathrm{Re}$ & Hoja & Infusión \\
\hline Lamiaceae & Origanum vulgare $\mathrm{L}$. & Sacha oregano & Herbáceo & Introducido & Silvestre & $\operatorname{Re}$ & Hoja & Infusión \\
\hline Lamiaceae & Salvia occidentalis $\mathrm{Sw}$. & Salvia azul & Herbáceo & Nativo & Silvestre & $\mathrm{Pa}$ & Hoja & Infusión \\
\hline Lamiaceae & Melissa officinalis $\mathrm{L}$. & Toronjil & Herbáceo & Nativo & Silvestre & $\mathrm{Ci}$ & Hoja & Infusión \\
\hline Lauraceae & $\begin{array}{l}\text { Persea americana Mill. } \\
\text { Mentzelia cordifolia Dombey ex }\end{array}$ & Palta & Herbáceo & Introducido & Cultivado & $\mathrm{Ge}, \mathrm{En}, \mathrm{Mu}$ & $\begin{array}{l}\text { Semilla } \\
\text { Planta }\end{array}$ & Decocción \\
\hline Loasaceae & $\begin{array}{l}\text { Urb. \& Gilg } \\
\text { Psittacanthus chanduyensis }\end{array}$ & Ancusacha & Herbáceo & Nativo & Silvestre & $\mathrm{Ge}$ & entera & Infusión \\
\hline Loranthaceae & Eichler & Suelda con suelda & Parásito & Introducido & Silvestre & $\begin{array}{c}\mathrm{Mu} \\
\mathrm{Di}, \mathrm{Ge}, \mathrm{Mu}, \mathrm{Pa}, \mathrm{En},\end{array}$ & Hoja & Emplasto \\
\hline Lytraceae & Punica granatum $\mathrm{L}$. & Granadilla & Arbustivo & Nativo & Cultivado & $\mathrm{Pi}$ & Fruto & Decocción \\
\hline Malvaceae & Gossypium barbadense $\mathrm{L}$. & Algodón & Herbáceo & Introducido & Cultivado & $\mathrm{Nn}$ & Semilla & Decocción \\
\hline Malvaceae & Malva sylvestris $\mathrm{L}$. & Malva blanca & Herbáceo & Introducido & Silvestre & $\mathrm{Ci}, \mathrm{Ge}$ & Hoja & Infusión \\
\hline Moraceae & Morus nigra $\mathrm{L}$. & Mora & Herbáceo & Nativo & Silvestre & $\mathrm{Re}, \mathrm{Ge}$ & Fruto & Decocción \\
\hline Moraceae & Morus alba $\mathrm{L}$. & Zarza mora & Herbáceo & Introducido & Silvestre & $\mathrm{Re}$ & Hoja & Infusión \\
\hline Musaceae & $\begin{array}{l}\text { Musa acuminata Colla } \\
\text { Myrica pubescens } \text { H. \& B. ex }\end{array}$ & Platano & Herbáceo & Introducido & Cultivado & $\mathrm{Pa}$ & Savia & Infusión \\
\hline Myricaceae & Wild. & Laurel & Arbustivo & Nativo & Cultivado & $\mathrm{Ge}, \mathrm{En}, \mathrm{Nn}$ & Tallo & $\begin{array}{l}\text { Decocción } \\
\text { Infusión, }\end{array}$ \\
\hline Myrtaceae & Eucalyptus globulus Labill. & Eucalipto & Arbóreo & Introducido & Cultivado & $\mathrm{Re}$ & Hoja & Vapor \\
\hline Myrtaceae & Psidium guajava $\mathrm{L}$. & Guayaba & Herbáceo & Nativo & Cultivado & $\mathrm{Re}, \mathrm{Di}, \mathrm{Nn}$ & $\begin{array}{l}\text { Fruto } \\
\text { Planta }\end{array}$ & Decocción \\
\hline Onagraceae & Oenothera rosea L'Hér. ex Aiton & Palisangre & Herbáceo & Nativo & Silvestre & $\mathrm{Ci}$ & $\begin{array}{l}\text { entera } \\
\text { Planta }\end{array}$ & Infusión \\
\hline Phrymaceae & $\begin{array}{l}\text { Mimulus glabratus Kunth } \\
\text { Peperomia aff. chachapoyasensis }\end{array}$ & Ucururu & Herbáceo & Nativo & Silvestre & $\mathrm{Di}, \mathrm{Pa}$, En & entera & Infusión \\
\hline Piperaceae & C. DC. & Congona & Herbáceo & Nativo & Silvestre & $\mathrm{Ci}$ & Hoja & Infusión \\
\hline Piperaceae & Piper marginatum Jacq. & Matico & Herbáceo & Nativo & Silvestre & $\mathrm{Re}, \mathrm{Di}, \mathrm{Ge}$ & Hoja & Infusión \\
\hline Plantaginaceae & Plantago major $\mathrm{L}$. & Llanten & Herbáceo & Nativo & Silvestre & $\mathrm{Ge}, \mathrm{Mu}, \mathrm{Pa}, \mathrm{En}, \mathrm{Nn}$ & Hoja & Infusión \\
\hline Plantaginaceae & Plantago lanceolata $\mathrm{L}$. & Llanten suave & Herbáceo & Nativo & Silvestre & En & Hoja & Infusión \\
\hline Poaceae & Saccharum officinarum $\mathrm{L}$. & Caña de azucar & Herbáceo & Introducido & Cultivado & $\mathrm{Ge}$ & Tallo & Decocción \\
\hline Poaceae & Arundo donax $\mathrm{L}$. & Carrizo & Herbáceo & Introducido & Silvestre & $\mathrm{Nn}$ & $\begin{array}{l}\text { Hoja } \\
\text { Planta }\end{array}$ & Infusión \\
\hline Poaceae & Cortaderia nitida (Kunth) Pilg. & Cortadera & Herbáceo & Introducido & Silvestre & $\mathrm{Ci}, \mathrm{Pa}$ & entera & Decocción \\
\hline Poaceae & Cymbopogon citratus (DC.) Stapf & Hierba luisa & Herbáceo & Introducido & Cultivado & $\mathrm{Re}, \mathrm{Di}, \mathrm{Ne}$ & Hoja & Infusión \\
\hline Poaceae & Chusquea sp. & Jun jul & Herbáceo & Nativo & Silvestre & $\mathrm{Ci}, \mathrm{Mu}$ & Hoja & Infusión \\
\hline Poaceae & Zea mays $\mathrm{L}$. & Maiz blanco & Herbáceo & Introducido & Cultivado & $\mathrm{Ci}, \mathrm{Nn}$ & $\begin{array}{l}\text { Fruto } \\
\text { Planta }\end{array}$ & Decocción \\
\hline Polygonaceae & Rumex sp. & Mala hierba & Herbáceo & Introducido & Silvestre & $\mathrm{Di}$ & entera & Infusión \\
\hline Polygalaceae & Monnina salicifolia Ruiz \& Pav. & Warmu warmu & Arbustivo & Nativo & Silvestre & $\mathrm{Di}, \mathrm{Ge}, \mathrm{Mu}, \mathrm{Pa}, \mathrm{En}$ & $\begin{array}{l}\text { Hoja } \\
\text { Planta }\end{array}$ & Infusión \\
\hline Portulacaceae & Portulaca oleracea $\mathrm{L}$. & Verdolaga & Herbáceo & Introducido & Silvestre & En & entera & Decocción \\
\hline Polygonaceae & $\begin{array}{l}\text { Rheum officinale Baill. } \\
\text { Oreocallis grandiflora (Lam.) R. }\end{array}$ & Ruibarbo & Herbáceo & Nativo & Silvestre & $\mathrm{Mu}$ & Hoja & Emplasto \\
\hline Proteaceae & Br. & Par par & Herbáceo & Nativo & Silvestre & $\mathrm{Re}, \mathrm{Ge}, \mathrm{Mu}, \mathrm{En}$ & Hoja & Decocción \\
\hline Rosaceae & Prunus sp1. & Duraznillo & Arbustivo & Nativo & Silvestre & $\mathrm{Ci}$ & Fruto & Decocción \\
\hline Rosaceae & Prunus sp2. & Durazno & Arbóreo & Nativo & Cultivado & $\mathrm{Pa}, \mathrm{Nn}$ & Fruto & Decocción \\
\hline Rosaceae & Rosa centifolia $\mathrm{L}$. & Rosa blanca & Herbáceo & Introducido & Silvestre & $\mathrm{Ci}, \mathrm{Ge}$ & Hoja & Infusión \\
\hline Rubiaceae & Coffea arabica $\mathrm{L}$. & Café & Arbustivo & Introducido & Cultivado & $\mathrm{Ci}$ & Semilla & Decocción \\
\hline Rubiaceae & Cinchona sp. & Cascarilla & Arbóreo & Nativo & Silvestre & $\mathrm{Pa}, \mathrm{Ci}$ & Tallo & Decocción \\
\hline Rutaceae & Citrus limon (L.) Burm. f. & Limon & Arbustivo & Introducido & Cultivado & $\mathrm{Pa}, \mathrm{Nn}$ & $\begin{array}{l}\text { Fruto } \\
\text { Planta }\end{array}$ & Decocción \\
\hline Rutaceae & Ruta graveolens $\mathrm{L}$. & Ruda & Arbustivo & Introducido & Silvestre & $\mathrm{Pa}, \mathrm{Nn}$ & entera & Infusión \\
\hline Salicaceae & Populus sp. & Alamo & Arbóreo & Nativo & Silvestre & $\mathrm{Nn}$ & Hoja & Decocción \\
\hline Sapindaceae & Dodonaea viscosa Jacq. & Chamana & Arbustivo & Nativo & Silvestre & $\mathrm{Ge}, \mathrm{Mu}, \mathrm{Nn}$ & Hoja & Infusión \\
\hline Solanaceae & Capsicum sp. & Aji de monte & Arbustivo & Nativo & Cultivado & $\mathrm{Pi}$ & Fruto & Emplasto \\
\hline
\end{tabular}




\begin{tabular}{|c|c|c|c|c|c|c|c|c|}
\hline Solanaceae & Brugmansia arborea (L.) Lagerh. & Floripondio & Arbustivo & Nativo & Silvestre & $\mathrm{Mu}$ & Hoja & $\begin{array}{c}\text { Emplasto, } \\
\text { Vapor }\end{array}$ \\
\hline Solanaceae & Solanum nigrum $\mathrm{L}$. & Hierba mora & Arbustivo & Nativo & Silvestre & $\mathrm{Di}, \mathrm{Ci}, \mathrm{Pa}$ & Hoja & Infusión \\
\hline Solanaceae & Cestrum auriculatum L'Hér. & Hierba santa & Herbáceo & Nativo & Silvestre & $\mathrm{Re}, \mathrm{Pa}, \mathrm{Nn}$ & Hoja & Infusión \\
\hline Solanaceae & Solanum tuberosum $\mathrm{L}$. & Papa & Herbáceo & Nativo & Cultivado & $\mathrm{Ge}, \mathrm{Pa}$ & Raíz & $\begin{array}{l}\text { Decocción } \\
\text { Infusión, }\end{array}$ \\
\hline Solanaceae & Nicotiana tabacum $\mathrm{L}$. & Tabaco & Arbustivo & Nativo & Cultivado & $\mathrm{Nn}$ & Hoja & Vapor \\
\hline Solanaceae & Physalis peruviana $\mathrm{L}$. & Tomate de monte & Herbáceo & Nativo & Silvestre & $\mathrm{Pa}$ & Fruto & Decocción \\
\hline $\begin{array}{l}\text { Solanaceae } \\
\text { Taxon }\end{array}$ & Physalis pubescens $\mathrm{L}$. & Tomatillo & Herbáceo & Nativo & Silvestre & $\mathrm{Pa}$ & Fruto & Decocción \\
\hline $\begin{array}{l}\text { Indeterminado sp1 } \\
\text { Taxon }\end{array}$ & Taxon Indeterminado sp1 & Pakakuna & Herbáceo & Nativo & Silvestre & $\mathrm{Mu}$ & Hoja & Emplasto \\
\hline $\begin{array}{l}\text { Indeterminado sp2 } \\
\text { Taxon }\end{array}$ & Taxon Indeterminado sp2 & Quintetuyo & Herbáceo & Nativo & Silvestre & $\mathrm{Mu}$ & Hoja & Emplasto \\
\hline Indeterminado sp3 & Taxon Indeterminado sp3 & Rumbsagua & Herbáceo & Nativo & Silvestre & $\mathrm{Ci}$ & Hoja & Infusión \\
\hline Typhaceae & Typha angustifolia $\mathrm{L}$. & Guineo & Herbáceo & Introducido & Silvestre & $\mathrm{Ci}, \mathrm{Ge}, \mathrm{Pa}, \mathrm{En}$ & $\begin{array}{l}\text { Hoja } \\
\text { Planta }\end{array}$ & Infusión \\
\hline Urticaceae & Pilea microphylla (L.) Lieberman & Contrayerba & Herbáceo & Nativo & Silvestre & $\mathrm{Ci}$ & entera & Infusión \\
\hline Urticaceae & Urtica magellanica Juss. ex Poir. & Ishanguilla & Herbáceo & Nativo & Silvestre & $\mathrm{Re}, \mathrm{Ci}, \mathrm{Mu}$ & Hoja & Baño \\
\hline Urticaceae & Urtica sp. & Ortiga & Herbáceo & Nativo & Silvestre & $\mathrm{Mu}$ & Hoja & Baño \\
\hline Verbenaceae & Verbena litoralis Kunth & Verbena & Herbáceo & Nativo & Silvestre & $\mathrm{Pa}, \mathrm{En}, \mathrm{Pi}, \mathrm{Nn}$ & Hoja & Infusión \\
\hline
\end{tabular}

(*)Sub-categorías de uso medicinal: Di: Trastornos del sistema digestivo, Pi: Afecciones cutáneas y subcutáneas, Vi: Trastornos de los ojos y anexos, Oi: Trastornos del oído y procesos mastoideos, Ci: Trastornos del sistema circulatorio, Ge: Trastornos del sistema genito-urinario, Mu: Trastornos del sistema musculo esquelético, Ne: Trastornos del sistema nervioso, Re: Trastornos del sistema respiratorio, En: Trastornos endocrinos, nutricionales y metabólicas, Pa: Dolencias infecciosas y parasitarias y Nn: Síntomas y afecciones clínicamente no clasificado.
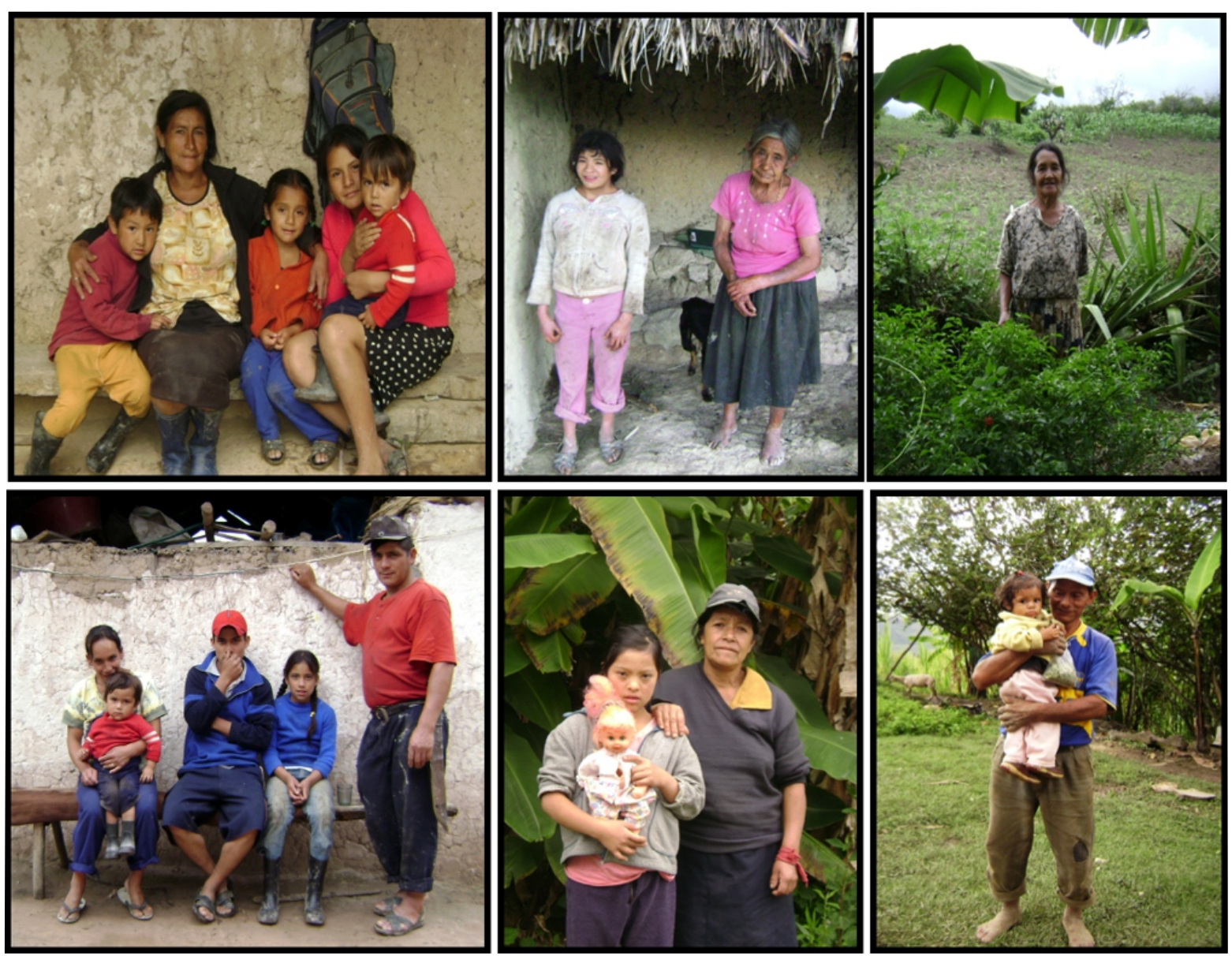

Figura 4. Informantes claves de las comunidades de San Antonio, Pencabamba y Lumac Urco, Chachapoyas, Amazonas, Perú. 
Dentro de los trastornos médicos más tratados con plantas medicinales en las comunidades estudiadas se destacan los: trastorno genito-urinario $(\mathrm{FCI}=0.65)$, trastorno respiratorio $(\mathrm{FCI}=0.58)$ y trastorno digestivo $(\mathrm{FCI}=0.55)$ (Figura 5), lo cual corrobora el amplio uso de plantas medicinales en el tratamiento de afecciones menores, cotidianas y culturales (Bussmann \& Glenn, 2010; Castillo-Vera, Cochachin, \& Albán, 2017).

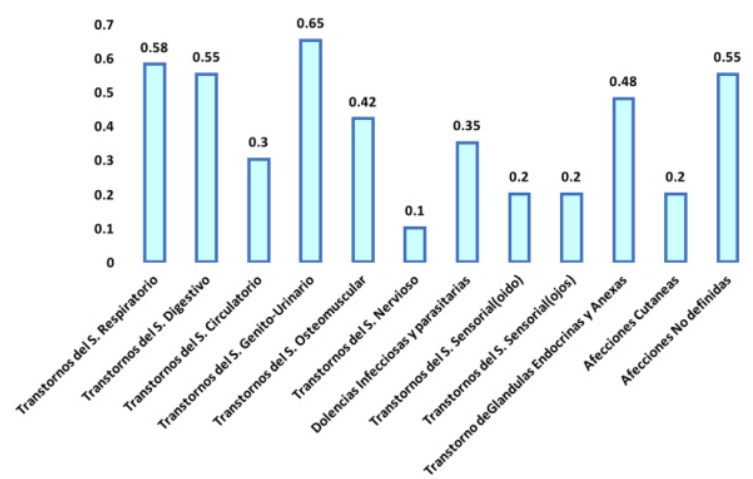

r ıgura 5. Sub-categorías de uso etnomedicinal. (Números indican valores de FCI).

Dentro de las especies etnomedicinales más importantes para las comunidades estudiadas se destacan: "hierba santa" Cestrum auriculatum L'Hér. $(\mathrm{IR}=1.75)$, "pie de perro" Desmodium uncinatum (Jacq.) DC. (IR=1.62), "Chishka" Baccharis tricuneata (L. f.) Pers. ( $\mathrm{IR}=1.62)$ "lancetilla" Alternanthera porrigens (Jacq.) Kuntze (IR=1.5). Asimismo, las partes más usadas de las especies etnomedicinales fueron: hoja $(49.2 \%)$, planta entera (24.2\%), fruto (12.1\%), semilla (5.6\%), tallo (4.8\%), raíz (3.2\%) y exudados (0.8\%) (Figura 6$)$.

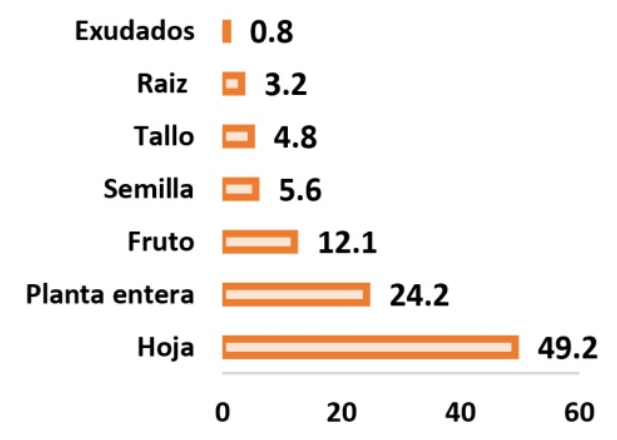

Figura 6. Principales partes usada de las especies etnomedicinales.

Se registraron 720 indicaciones sobre el uso y la preparación de las especies etnomedicinales en la zona de estudio, las cuales se dan principalmente: por infusión (62\%), decocción (28\%), emplasto (5\%), vapor (3\%), baño (2\%) (Figura 7$)$.

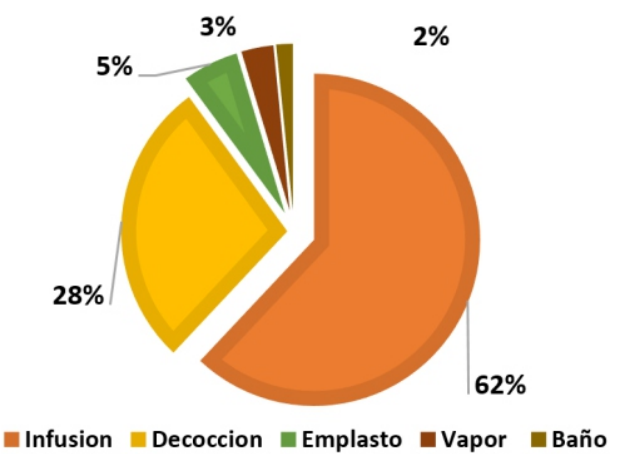

Figura 7. Principales formas de preparación de las especies etnomedicinales.

Finalmente, nuestro análisis del índice de Shannon $\left(\mathrm{H}^{\prime}\right)$ modificado a estudios etnobotánicos, nos demuestra que todas las comunidades estudiadas mantienen un buen nivel de conocimiento cultural (Tabla 3) en comparación a otros estudios etnobotánicos desarrollados en Sudamérica (Begossi, 1996; Christo et al., 2006).

Tabla 3. Valores del índice $\mathrm{H}^{\prime}$ en las comunidades estudiadas.

\begin{tabular}{cc}
\hline Comunidades & Índice $\mathbf{H}^{\prime}(\mathbf{b a s e} \mathbf{e})$ \\
\hline San Antonio & 5.5 \\
\hline Quipachacha & 5.2 \\
\hline Pencapampa & 4.6 \\
\hline Lucma Urco & 4.5 \\
\hline
\end{tabular}

\section{CONCLUSIONES}

En las comunidades estudiadas el uso de plantas medicinales constituye parte esencial de su vida cotidiana como lo demuestran los altos valores del índice $\mathrm{H}^{\prime}$, el alto número de especies medicinales reportadas y el amplio espectro de trastornos médicos para los cuales son usadas. Asimismo, la naturaleza herbácea de la mayoría de especies etnomedicinales reportadas en la investigación las muestran como un componente frágil ante perturbaciones de sus hábitats producto de acción antrópica.

Cuantificación y corrobación química de las plantas medicinales reportadas en este estudio, evaluación de la vulnerabilidad de sus poblaciones naturales y análisis de transmisión de conocimientos tradicionales hacia las nuevas generaciones son posibles temáticas a desarrollar en el futuro. 


\section{REFERENCIAS}

Albán, J. A. (1998). Etnobotánica y conservación en la comunidad andina de Pamparomas, Huaylas, Ancash. Perú (Tesis MSc en Etnobotanica). Facultad de Ciencias Biologicas-Universidad Nacional Mayor de San Marcos, Lima, Perú.

Alva, R. A., Quezada, M. R., \& Rodríguez, E. P. (2016). Metabolitos secundarios de mezcla de plantas medicinales con acción antibacterial sobre microorganismos causantes de infección puerperal en la provincia de Chachapoyas. Pueblo Continente, 25(2), 61-69.

APG. (1998). An ordinal classification for the families of flowering plants. Annals of the Missouri Botanic Garden, 85, $531-553$. https://doi.org/10.2307/2992015

APG II. (2003). An update of the Angiosperm Phylogeny Group classification for the ordens and families of flowering plants: APG II. Botanical Journal of the Linnean Society, 141, 339-436.

APG III. (2009). An update of the Angiosperm Phylogen Group classification for the orders and families of flowering plants: APG III. Botanical Journal of the Linnean Society, 161, 105-121.

APG IV. (2016). An update of the Angiosperm Phylogeny Group classification for the orders and families of flowering plants: APG IV. Botanical Journal of the Linnean Society, 181(1), 1-20.

Balick, M. J. (1990). Ethnobotany and the indentification of therapeutic agents from the rainforest. (D. Chadwick \& J. Marsh, Eds.), Ciba Foundation Symposium.

Begossi, A. (1996). Use of ecological methods in ethnobotany: Diversity indices. Economic Botany, 50(3), 280-289.

Bennett, B., \& Prance, G. (2000). Introduced plants in the indigenous Pharmacopoeia of Northern South America. Economic Botany, 54(1), 90-102.

Berkes, F., Colding, J., \& Folke, C. (2000). Rediscovery of traditional ecological knowledge as adaptive management. Ecological Adaptations, 10(October), 1251-1262.

Bernard, H. R. (2006). Research methods in Anthropology: Qualitative and Quantitative Approaches. Psychological Methods (AltaMira P).

Boster, J. S. (1986). Exchange of Varieties and Information Between Aguaruna Manioc Cultivators.
American Anthropologist, 88(2), 428-436.

Brako, L., \& Zarucchi, J. L. (1993). Catalogue of the Flowering Plants and Gymnosperms of Peru. Monographs in Systematic Botany (Vol. 45). Missouri Botanical Garden.

Bussmann, R. W., \& Glenn, A. (2010). Medicinal plants used in Peru for the treatment of respiratory disorders in Complementary Medicine and the Pan American Health allopathic medicine in clinics and hosp. Thymus, 17(2), 331-346.

Bussmann, R. W., \& Sharon, D. (2006). Traditional medicinal plant use in Northern Peru: tracking two thousand years of healing culture. Journal of Ethnobiology and Ethnomedicine, 2, 47.

Case, R., Pauli, G., \& Soejarto, D. D. (2005). Factors in maintaining indigenous knowledge among ethnic communities of Manus Island. Economic Botany, 59(4), 356-365.

Castillo-Vera, H., Cochachin, E., \& Albán, J. (2017). Plantas comercializadas por herbolarios en el mercado del distrito de Cajabamba (Cajamarca , Perú). Boletin Latinoamericano Y Del Caribe de Plantas Medicinales Y Aromaticas, 16(3), 303-318.

Cerrate, E. (1969). Manera de preparar plantas para un Herbario. , , No. 1. 10 pp. Revista Del Museo de Historia Natural de La UNMSM., (Serie de Divulgación), 1-10.

Christo, A. G., Guedes-Bruni, R. R., \& FonsecaKruel, V. S. (2006). Uso de recursos vegetais em comunidades rurais limítrofes à reserva biológica de Poço das Antas, Silva Jardim, Rio de Janeiro: Estudo de caso na Gleba Aldeia Velha. Rodriguésia, 57(3), 519-542.

De-la-Cruz, H., Vilcapoma, G., \& Zevallos, P. A. (2007). Ethnobotanical study of medicinal plants used by the Andean people of Canta, Lima, Peru. Journal of Ethnopharmacology, 111(2), 284-294.

Encarnación, F., \& Zárate, R. (2010). Vegetación. In Proyecto para zonificacion ecologica y economica del departamento de Amazonas (IIAP y el, p. 38). Iquitos-Peru.

Fajardo, J., Lessmann, J., Bonaccorso, E., Devenish, C., \& Muñoz, J. (2014). Combined use of systematic conservation planning, species distribution modelling, and connectivity analysis reveals severe conservation gaps in a megadiverse country (Peru). PLoSONE, 9(12), e114367.

Garcia, F. (2008). Impacto antropico en las plantas medicinales nativas del departamento de Amazonas, 
Peru. Universidad Nacional de Trujillo.

Gentry, A. H. (1982). Neotropical Floristic " Diversity : Phytogeographical Connections Between Central and South America, Pleistocene Climatic Fluctuations, or an Accident of the Andean Orogeny ? , 2. Annals of the Missouri Botanical Garden, 69(3), 557-593.

Grados Vasquez, M., \& Peláez, F. (2014). Especies vegetales utilizadas por pobladores de Berlín, Bagua Grande (Amazonas, Perú) 2011-2012. Rebiolest, 2(2), e36.

Lawrence, A., Phillips, O. L., Ismodes, A. R., Lopez, M., Rose, S., Wood, D., \& Farfan, A. J. (2005). Local values for harvested forest plants in Madre de Dios, Peru: Towards a more contextualised interpretation of quantitative ethnobotanical data. Biodiversity and Conservation, 14(1), 45-79.

McClatchey, W. C., Mahady, G. B., Bennett, B. C., Shiels, L., \& Savo, V. (2009). Ethnobotany as a pharmacological research tool and recent developments in CNS-active natural products from ethnobotanical sources. Pharmacology \& Therapeutics, 123(2), 239-254.

Monigatti, M., Bussmann, R. W., \& Weckerle, C. S. (2013). Medicinal plant use in two Andean communities located at different altitudes in the Bolívar Province, Peru. Journal of Ethnopharmacology, 145(2), 450-464.

Phillips, O., Gentry, A. H., Reynel, C., Wilkin, P., Galvez-Durand, C., \& Galvez-Durand, B. C. (1994). Quantitative ethnobotany and Amazonian conservation. Conservation Biology, 8(1), 225-248. Reyes-García, V., Vadez, V., Tanner, S., Huanca, T., Leonard, W. R., \& Mcdade, T. (2006). Measuring what people know about the environment: a review of quantitative studies. Tsimana' Amazonian Panel Study Working Paper.

Scott Atran, Douglas Medin, Norbert Ross, Elizabeth Lynch, Valentina Vapnarsky, Edilberto Ucan Ek', ... Michael Baran. (2002). Folkecology, Cultural Epidemiology, and the Spirit of the Commons: A Garden Experiment in the Maya Lowlands, 1991-2001. Current Anthropology, 43(3), 421-450.

SERNARP. (2014). Areas de Conservacion Privada. Lima - Perú.

Torre, L. D. La, Muriel, P., Balslev, H., De la Torre, L., Muriel, P., \& Balslev, H. (2006). Etnobotánica en los Andes del Ecuador. Botánica Económica de Los Andes Centrales., (January 2006), 246-267.
Trotter, R. T., \& Logan, M. H. (1986). Informant Consensus: A new approach for identifying potentially effective medicinal plants. In Plants in Indigenous Medicine and Diet: Biobehavioural approaches (Etkin, N., pp. 91-112). Bedford Hills, NY.: Redgrave Publishers.

WHO. (2016). International Statistical Classification of Diseases and Related Health Problems 10th Revision (ICD-10)-WHO Version for 2016. 